\title{
The sympathetic skin response in the shoulder-hand syndrome complicating tetraplegia
}

\author{
ML Aisen ${ }^{1}, \mathrm{~J} \mathrm{Stallman}^{1}$ and PS Aisen ${ }^{2}$ \\ ${ }^{1}$ The Burke Rehabilitation Center, 785 Mamaroneck Avenue, White Plains, New York 10605; ${ }^{2}$ Department of \\ Medicine, Mount Sinai Medical Center, One Gustave L Levy Place, New York, New York 10029, USA
}

To determine the utility of the sympathetic skin response (SSR) in studying sympathetic outflow in cervical spinal cord injury (CSCI) patients who develop features of reflex sympathetic dystrophy, we studied seven consecutive CSCI patients with the shoulder-hand syndrome (SHS), and seven patients with similar injuries but without the manifestations of the SHS. The mean SSR amplitude was more than three times greater in patients with the SHS, a statistically significant difference. We restudied six patients with a SHS after symptoms had improved following steroid treatment: the mean amplitude fell significantly, approaching the mean for the control group. When pain was asymmetric, the SSR amplitude was higher in the arm with greater pain. Four of the seven SHS patients demonstrated an unusual pattern of spontaneous cyclical electrical activity during a period of severe pain. We conclude that a SHS complicating CSCI is associated with increased amplitude of the SSR, supporting the theory that sympathetic hyperactivity is important in the pathophysiology of reflex sympathetic dystrophy in this setting.

Keywords: sympathetic skin response; shoulder-hand syndrome; reflex sympathetic dystrophy; tetraplegia; spinal cord injury

\section{Introduction}

The role of the sympathetic nervous system in reflex sympathetic dystrophy (RSD) and other chronic pain disorders remains uncertain. The diagnosis of RSD is made on clinical grounds; the characteristic features include: pain (usually of 'burning' quality) with tenderness, edema, vasomotor instability, trophic changes of the skin and nails, osteoporosis on radiographs, and increased uptake on radionuclide bone scans. ${ }^{1,2}$ While most investigators attribute pathophysiological significance to sympathetic dysfunction in RSD, only a portion of patients respond to sympathetic blockade. Though it has long been assumed that features of RSD are mediated by increased sympathetic outflow, conflicting evidence has been reported. ${ }^{3,4}$ While it may be clinically useful to categorize chronic pain patients into two groups based on response to sympathetic blockade, patients meeting strict criteria for RSD may fall into either category. ${ }^{5}$ Recent studies involving double-blind infusions of sympathetic agonist and antagonist drugs to chronic pain patients call into question the concept of 'sympathetically maintained pain'. 6,7

A method of measurement of sympathetic nerve function in the extremities would be useful in characterizing the role of sympathetic dysfunction in RSD and other pain disorders. The sympathetic skin response (SSR), a technique involving measurement of voltage change across the palm following electrical stimulation, is a candidate for this purpose. The SSR can be measured with standard electromyographic equipment, and has been useful in the study of sympathetic function in various peripheral nerve diseases. ${ }^{8,9}$

The shoulder-hand syndrome (SHS), a form of RSD involving the upper extremities, is prevalent in patients undergoing rehabilitation following traumatic cervical spinal cord injury. ${ }^{10-12}$ The common occurrence of the SHS in this setting may be related to the generalized autonomic dysfunction that is characteristic of tetraplegia. The SSR can be measured in tetraplegic individuals, and demonstrates low amplitudes and prolonged latencies compared to normal controls, with an inverse correlation between amplitude and severity of injury. ${ }^{13}$

In this paper, we report and discuss the results of SSR studies performed on seven patients with traumatic cervical spinal cord injury who developed a SHS.

\section{Methods}

Seven consecutive cervical spinal cord injury patients of The Burke Rehabilitation Hospital who developed SHS were selected for neurophysiological studies. For comparison, seven patients with similar injuries but without manifestations of a SHS were also studied. The experimental protocol was approved by the institution's committee for human rights in research, and informed consent was obtained from each participant.

In each case, the diagnosis of the SHS was based 
on the presence of diffuse upper extremity pain with a 'burning' quality, edema, osteoporosis evident on radiographs, and, in the majority of the subjects, dystrophic skin and nail changes. Each patient met the criteria for probable RSD proposed by Kozin et al ${ }^{2}$

The patients were tested in the inpatient ward sitting upright or slightly inclined in a wheelchair. They were asked to keep their eyes closed for the duration of the session. The ambient temperature was maintained at 73 degrees Fahrenheit. For patients with a SHS, the arm with greater pain was studied. The SSR was recorded with a Dantec Counterpoint Model Mk2 (Dantec, Skovlunde, Denmark). Using electrode coupling gel, a Dantec $13120 \mathrm{Ag} / \mathrm{AgCl}$ ECG surface electrode was placed on the palmar surface, and a similar reference electrode was placed in the center of the dorsal surface. Dantec 13L36 stimulation electrodes were attached with surgical tape to $\mathrm{C} 3$ and $\mathrm{T} 8$ dermatomes corresponding to sensory levels above and below the level of injury. After recording spontaneous activity, a square wave electrical stimulus of $20.0 \mathrm{Ma}$ was applied for a period of $0.2 \mathrm{~ms}$, and the response was recorded for $8 \mathrm{~s}$. To avoid habituation, stimuli were applied to the $\mathrm{C} 3$ or $\mathrm{T} 8$ site in a seemingly random sequence at a frequency of no greater than one stimulation per min.

Measurements of amplitude and latency were obtained from subsequent analysis of individual traces. Since waveforms vary considerably between and within subjects, every tracing was independently interpreted by two investigators. The latency was defined as the time from stimulation to the onset of the sympathetic response. The amplitude was defined as the absolute distance between the initial negative, then positive deflection. All tracings with clear SSRs were scored for amplitude and latency. Tracings with no recognizable response, or with other electrical activity (ie muscle movement) were disregarded. The three tracings with the largest amplitudes were selected for each stimula- tion site, and used to calculate the mean amplitude and latency.

Statistical analysis was performed with StatView II on a Macintosh II computer. A one-tailed, paired $t$ test was used to compare the mean amplitudes and latencies.

\section{Results}

The clinical characteristics of the SHS patients and the control group are shown in Table 1. The interval between injury and SSR examination was longer in the control group, but SSR amplitude in tetraplegic patients was unrelated to time since injury. ${ }^{13}$

Figure 1 shows the SSR amplitudes for all the subjects. The mean amplitude was more than three times greater in patients with SHS, a statistically significant difference.

Six patients with a SHS were restudied after symptoms had improved following steroid treatment. As shown in Figure 2, the mean amplitude fell significantly, approaching the mean for the control group.

Symptoms of the SHS are usually bilateral. ${ }^{2,12}$ When pain was asymmetric, the SSR amplitude was higher in the arm with greater pain. Figure 3 shows bilateral measurements before and after treatment in a patient with asymmetric pain. The left hand, which was the more painful, had a greater amplitude before treatment; after resolution of symptoms, the SSR response was symmetric.

Four of the seven SHS patients demonstrated an unusual pattern of spontaneous electrical activity during a period of severe pain, as depicted in Figure 4. Prior to any stimulation, both tracings demonstrated large amplitude sinusoidal deflections with a frequency of about $1.6 \mathrm{hz}$. This pattern has not been found during repeated testing of 16 patients with CSCI without SHS.

Table 1 Clinical Characteristics

\begin{tabular}{|c|c|c|c|c|c|c|}
\hline Patient & Sex & Age & $\begin{array}{c}\text { Injury } \\
\text { level }\end{array}$ & $\begin{array}{l}\text { Frankel } \\
\text { score }^{14}\end{array}$ & $\begin{array}{l}\text { Interval between } \\
\text { injury and onset of } \\
\text { shoulder-hand } \\
\text { syndrome }\end{array}$ & $\begin{array}{l}\text { Interval between } \\
\text { injury and SSR } \\
\text { examination }\end{array}$ \\
\hline \multicolumn{7}{|c|}{ Tetraplegic controls } \\
\hline 1 & $\mathbf{M}$ & 27 & $\mathrm{C} 5$ & A & - & 5 months \\
\hline 2 & $\mathbf{M}$ & 40 & $\mathrm{C} 7$ & $\mathrm{C}$ & - & 7 years \\
\hline 3 & $\mathbf{M}$ & 36 & C6 & A & - & 12 years \\
\hline 4 & $\mathbf{M}$ & 68 & $\mathrm{C} 5$ & $\mathrm{D}$ & - & 5 years \\
\hline 5 & $\mathbf{M}$ & 30 & $\mathrm{C} 5$ & A & - & 3 years \\
\hline 6 & $\mathrm{~F}$ & 68 & $\mathrm{C} 2$ & $\mathrm{D}$ & - & 8 months \\
\hline 7 & $\mathbf{M}$ & 62 & $\mathrm{C} 3$ & $\mathrm{D}$ & - & 3 years \\
\hline \multicolumn{7}{|c|}{ Tetraplegic subjects with shoulder-hand syndrome } \\
\hline 1 & $\mathrm{M}$ & 55 & $\mathrm{C} 5-6$ & $\mathrm{C}$ & 51 days & 2 months \\
\hline 2 & $\mathbf{M}$ & 17 & $\mathrm{C} 5$ & A & 163 days & 5 months \\
\hline 3 & $\mathbf{M}$ & 33 & $\mathrm{C} 5-6$ & A & 14 days & 6 months \\
\hline 4 & $\mathrm{~F}$ & 50 & $\mathrm{C} 7$ & $\mathrm{D}$ & 6 days & 2 months \\
\hline 5 & $\mathbf{M}$ & 70 & C6 & $\mathrm{C}$ & 8 days & 2 months \\
\hline 6 & $\mathrm{~F}$ & 43 & $\mathrm{C} 5$ & A & 114 days & 5 months \\
\hline 7 & $\mathbf{M}$ & 70 & C6 & $\mathrm{D}$ & 25 days & 3 months \\
\hline
\end{tabular}




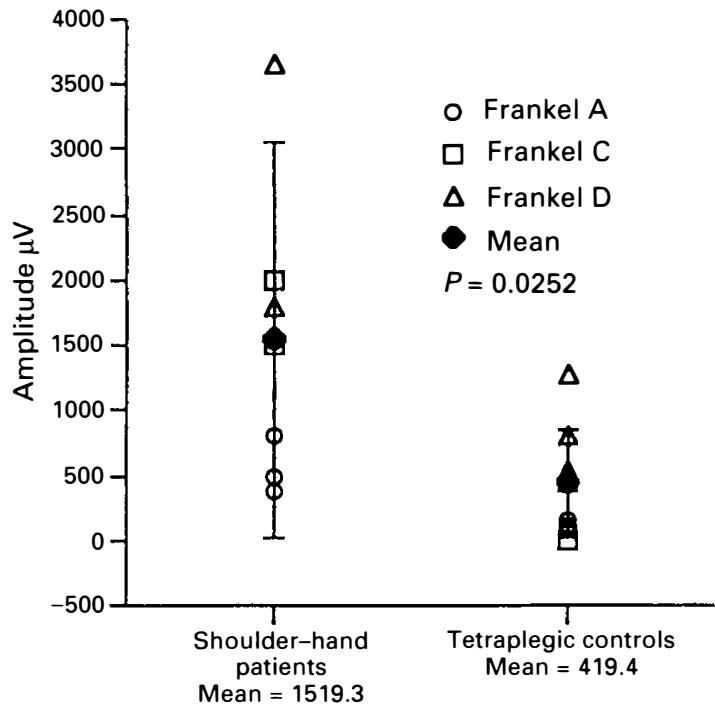

Figure 1 Sympathetic skin response amplitudes in tetraplegic subjects with and without shoulder-hand syndrome

It is not specific to the SHS: we have seen this response in one normal subject during a period of heightened anticipation prior to painful stimulus.

\section{Discussion}

These studies demonstrate that the SHS complicating CSCI is associated with increased amplitude of the SSR. This lends support to the theory that sympathetic hyperactivity is important in the pathophysiology of reflex sympathetic dystrophy in this setting.

A central question in the study of RSD is whether sympathetic outflow is increased in the affected limbs. Decreased skin temperature has been attributed to vasoconstriction mediated by increased sympathetic activity, but hypersensitivity of vascular receptors to

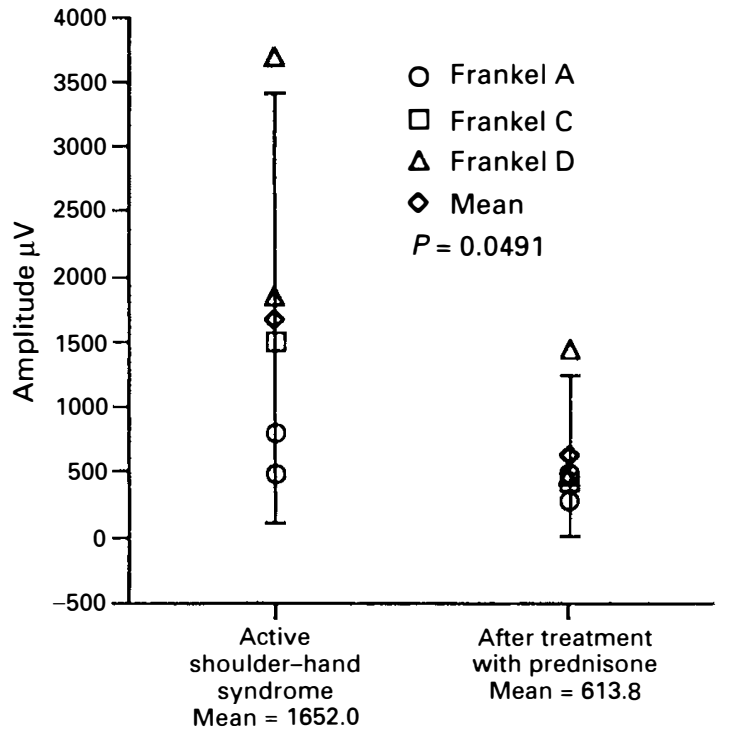

Figure 2 Sympathetic skin response amplitudes in tetraplegic subjects with shoulder-hand syndrome before and after treatment with prednisone

circulating catecholamines is an alternative explanation. ${ }^{15,16}$ A recent report of an unusual patient with RSD confined to the ulnar aspect of one hand demonstrated no sympathetic hyperactivity by microneurography and SSR recordings despite marked cutaneous vasoconstriction. ${ }^{4}$ Reduced catecholamine levels in the affected compared to unaffected limbs of RSD patients reported in another study support the receptor supersensitivity hypothesis. ${ }^{3}$

The SSR measures sweat gland activation and thus cannot definitively distinguish increased sympathetic outflow from receptor supersensitivity. However, it is intriguing to hypothesize that the spontaneous cyclical activity shown in Figure 4 represents a reverberating, disinhibited sympathetic loop. We have previously
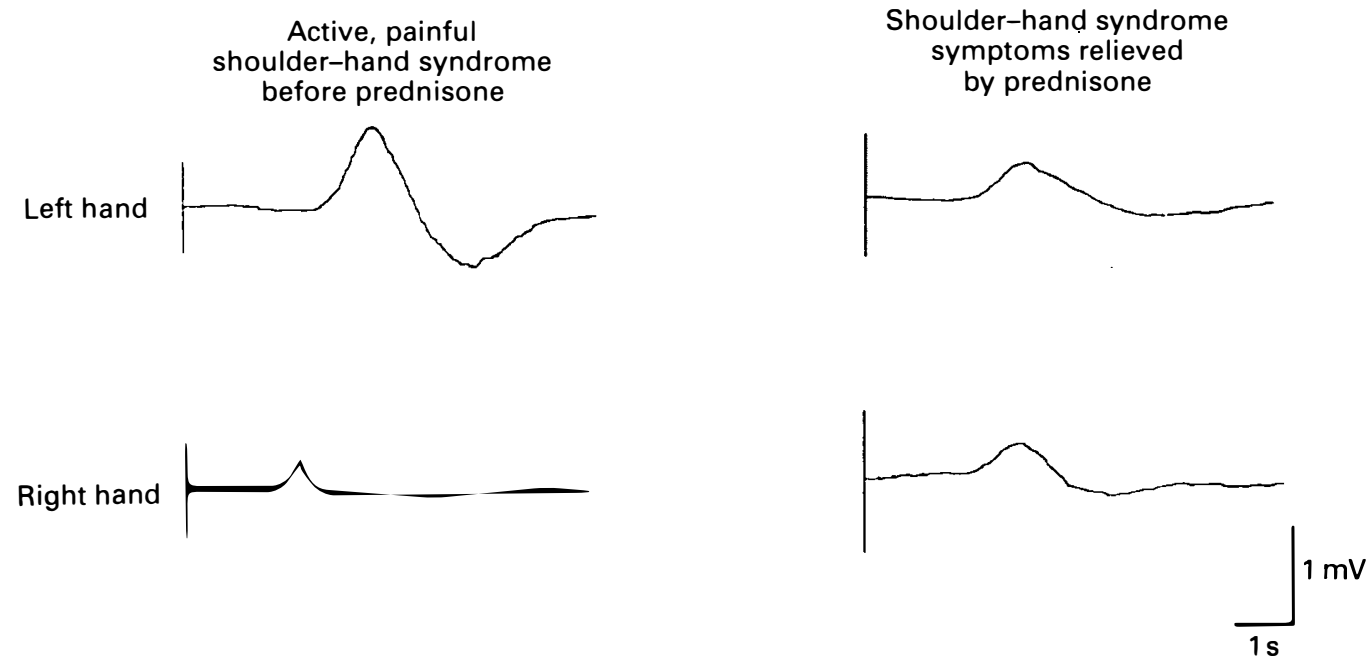

Figure 3 Bilateral sympathetic skin response measurements in a tetraplegic subject with shoulder-hand syndrome involving only the left limb 


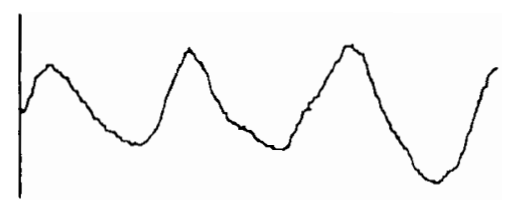

Patient 2

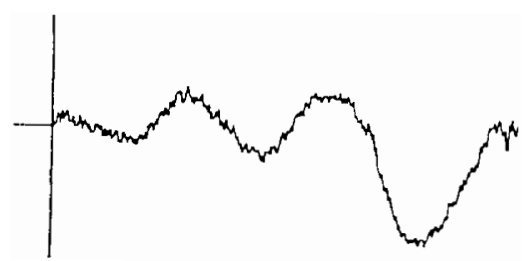

Patient 5

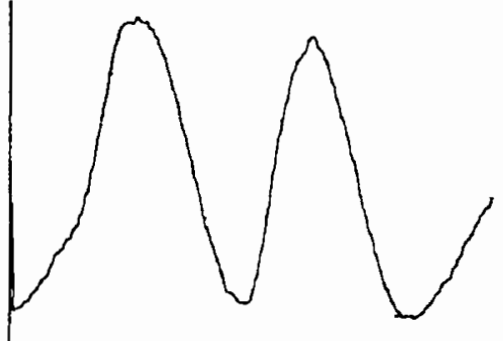

Patient 3

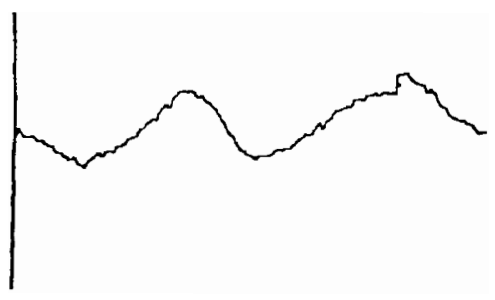

Patient 6

Figure 4 Spontaneous rhythmic electrical activity seen in four shoulder-hand syndrome subjects

reported that CSCI patients show diminished habituation of the SSR, consistent with impaired transmission of higher inhibitory influences across the injured spinal cord. ${ }^{13}$ Additional studies utilizing simultaneous SSR, microneurography and plasma catecholamine measurements would clarify the relative contribution of increased outflow and upregulation of receptors.

Despite this limitation, we suggest that the SSR is an underutilized, non-invasive tool which can provide important information to clarify the role of sympathetic pathways in RSD and other chronic pain disorders. For example, this technique may be used to determine the relationship between sympathetic activity and various clinical parameters in RSD, determine whether sympathetic hyperactivity predicts response to sympathetic blockade or other therapeutic modalities, and provide a measure of the adequacy of sympathetic blockade.

\section{References}

1 Schwartzman RJ. Reflex sympathetic dystrophy and causalgia Neurol Clin 1992; 10: 953-973.

2 Kozin $\mathrm{F}$ et al. The reflex sympathetic dystrophy syndrome (RSDS). III. Scintigraphic studies, further evidence for the therapeutic efficacy of systemic corticosteroids, and proposed diagnostic criteria. Am J Med 1981; 70: 23-30.

3 Drummond PD, Finch PM, Smythe GA. Reflex sympathetic dystrophy: the significance of differing plasma catecholamine concentrations in affected and unaffected limbs. Brain 1991; 114: 2025-2036.
4 Casale R, Elam M. Normal sympathetic nerve activity in a reflex sympathetic dystrophy with marked skin vasoconstriction. J Auton Nerv Syst 1992; 41: 215-220.

5 Davidoff G. The reflex sympathetic dystrophy syndrome, associated with traumatic myelopathy. Pain 1989; 39: 367.

6 Verdugo RJ, Ochoa JL. 'Sympathetically maintained pain'. I. Phentolamine block questions the concept. Neurology 1994; 44: 1003-1010.

7 Verdugo RJ, Campero M, Ochoa JL. Phentolamine sympathetic block in painful polyneuropathies. II. Further questioning of the concept of 'sympathetically maintained pain'. Neurology 1994; 44: 1010-1014.

8 Fagius J, Wallin BG. Sympathetic reflex activity and conduction velocities in patients with polyneuropathy. J Neurol Sci 1980; 47: 449-461.

9 Niakan E, Harati Y. Sympathetic skin response in diabetic peripheral neuropathy. Muscle Nerve 1987; 10: 711-716.

10 Gellman $\mathrm{H}$ et al. Reflex sympathetic dystrophy in cervical spinal cord injury patients. Clin Orthop 1988; 233: 126-131.

11 Cremer SA, Maynard F, Davidoff G. The reflex sympathetic dystrophy syndrome associated with traumatic myelopathy: report of 5 cases. Pain 1989; 37: 187-192.

12 Aisen PS, Aisen ML. Shoulder-hand syndrome in cervical spinal cord injury. Paraplegia 1994; 32: 588-592.

13 Aisen ML, Stallman J. The sympathetic skin response in quadriplegia. $J$ Neurorehabil (in press).

14 Frankel HL et al. The value of postural reduction in the initial management of closed injuries of the spine with paraplegia and tetraplegia. Paraplegia 1969; 7: 179-192.

15 Wallin G, Torebjork E, Hallin R. Preliminary observations on the pathophysiology of hyperalgesia in the causalgic pain syndrome. In: Zotterman Y (ed). Sensory Functions of the Skin in Primates: With Special Reference to Man. Oxford: Pergamon Press, 1976, pp 489-502.

16 Bennett GJ. The role of the sympathetic nervous system in painful peripheral neuropathy. Pain 1991; 45: 221-223. 